accumulation of longer polynucleosomal DNA fragments in circulating microparticles, and to autoantibody responses to microparticle-associated DNA and protein antigens.

Acknowledgments Supported by the NIH grants AR071703, AR070591 and AR069515, the Lupus Research Alliance, the Colton Center for Autoimmunity and the German Research Foundation (DFG).

\section{REGULATORY RNAS IN NEUROPSYCHIATRIC SYSTEMIC LUPUS ERYTHEMATOSUS (SLE)}

${ }^{1}$ Ilham A Muslimov, ${ }^{2}$ Ellen M Ginzler, ${ }^{1,3}$ Henri Tiedge*. ${ }^{1}$ The Robert F. Furchgott Center for Neural and Behavioral Sciences, Department of Physiology and Pharmacology: ${ }^{2}$ Division of Rheumatology, Department of Medicine; ${ }^{3}$ Department of Neurology; SUNY Downstate Health Sciences University, Brooklyn, New York 11203, USA

\subsection{6/lupus-2021-lupus21century.40}

Background Brain-specific Cytoplasmic (BC) RNAs operate as translational regulators at neuronal synapses. BC RNAs are delivered to synapto-dendritic sites of function by transport factors heterogeneous nuclear ribonucleoprotein A2 (hnRNP A2) and Pura. Dysregulation of BC RNA control has been associated with epilepsy and cognitive impairment. We hypothesized that structural motifs in BC RNAs can become targets of autoimmune reactivity in neuropsychiatric SLE.

Methods Sera were collected from patients with SLE, rheumatoid arthritis (RA), multiple sclerosis (MS), and from healthy subjects (HS). ${ }^{1}$ RNA-protein interactions were examined by electrophoretic mobility shift assays (EMSAs). Microinjection RNA transport analysis was performed with sympathetic neurons in primary culture. Sera or purified antibodies were injected i.v. into wild-type (WT) mice, in conjunction with i.p. injection of lipopolysaccharide to permeabilize the blood-brain barrier. $^{2}$

Results Autoantibodies against BC RNAs (anti-BC abs) were detected in a subset of SLE patient sera. Strength of SLE anti-BC autoimmune reactivities and occurrence of neuropsychiatric manifestations, in particular seizures, correlated

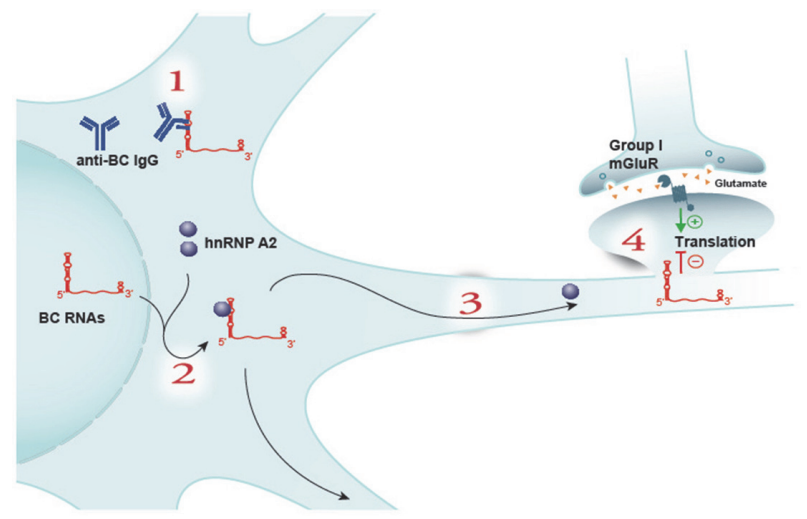

Abstract 902 Figure 1 SLE anti-BC abs: molecular - cellular mode of action. SLE anti-BC abs target transport-specifying DTEs in BC RNAs (here represented by rodent BC1 RNA; Scene 1). They prevent binding of hnRNP A2 (and Puro, not shown) to such DTEs (which occurs normally in the absence of SLE anti-BC abs; Scene 2). Dendritic delivery of BC RNAs (Scene 3) is thus compromised by SLE anti-BC abs. Lack of $B C$ RNAs at the synapse results in aberrant local translational control ((due to unbalanced metabotropic glutamate receptor stimulation of synaptic translation; Scene 4), causing network hyperexcitability and consequential phenotypic alterations. From ref. [1]. strongly (Spearman's $\mathrm{r}_{\mathrm{s}}=0.89, \mathrm{P}<0.0001$ ). Anti-BC abs were not detected in sera from RA or MS patients or in sera from HS. In human BC200 RNA, a noncanonical dendritic targeting element (DTE) is responsible for binding of transport factors hnRNP A2 and Pur $\alpha$ and for specifying delivery to synapto-dendritic domains. The same DTE is complexed by SLE anti-BC abs with high affinity and essentially irreversibly, in interactions that cause displacement of transport factors and inhibition of synapto-dendritic transport.

Lack of BC RNAs in neurons, either cell-wide or locally at the synapse, causes seizure susceptibility and cognitive impairment. ${ }^{3-5}$ We posited that introduction of SLE anti-BC IgGs into the brains of naïve WT mice, which causes BC RNA dendritic transport inhibition and thus depletion at the synapse, would result in analogous phenotypes. Indeed upon auditory stimulation, such mice succumbed to severe generalized tonic-clonic seizures (seizure rate 100\%, mortality 100\%). Mice injected with non-SLE IgGs (RA, MS, HS) never seized. Significantly, when SLE anti-BC IgGs were coinjected with human BC200 RNA, seizures did not materialize (Fisher's Exact Test, $\mathrm{P}<0.0001)$.

Conclusions Our data show that SLE anti-BC IgGs, isolated from sera of lupus patients with a history of seizures, cause severe seizures in animals. Seizures are completely prevented if SLE anti-BC IgGs are complexed with BC200 RNA. We propose that this approach may lend to the development of therapeutic interventions using BC200 decoys.

\section{REFERENCES}

1. Muslimov IA, lacoangeli A, Eom T, Ruiz A, Lee $M$, Stephenson S, Ginzler EM, Tiedge $H$. Neuronal BC RNA transport impairments caused by systemic lupus erythematosus autoantibodies. J Neurosci 2019;39:7759-7777.

2. Kowal C, Degiorgio LA, Lee JY, Edgar MA, Huerta PT, Volpe BT, Diamond B Human lupus autoantibodies against NMDA receptors mediate cognitive impairment. Proc Natl Acad Sci USA 2006;103:19854-19859.

3. Zhong J, Chuang SC, Bianchi R, Zhao W, Lee H, Fenton AA, Wong RK, Tiedge H. $\mathrm{BC} 1$ regulation of metabotropic glutamate receptor-mediated neuronal excitability. J Neurosci 2009;29:9977-9986.

4. Muslimov IA, Eom T, lacoangeli A, Chuang SC, Hukema RK, Willemsen R, Stefanov DG, Wong RKS, Tiedge $H$. BC RNA mislocalization in the fragile $X$ premutation. eNeuro 5. 2018.

5. lacoangeli A, Dosunmu A, Eom T, Stefanov DG, Tiedge $H$. Regulatory BC1 RNA in cognitive control. Learn Mem 2017;24:267-277.

Acknowledgments We thank Drs. Dimitre Stefanov and Jeremy Weedon for statistical consultation. Funding for this work was provided by the Rheumatology Research Foundation and by NIH grants DA026110 and NS046769 (HT).

\section{0 - Patient-reported outcomes}

\section{LONGITUDINAL CHANGES IN TYPE 2 SLE ACTIVITY}

${ }^{1}$ Amanda M Eudy*, ${ }^{1}$ Jennifer L Rogers, ${ }^{2}$ Daniel Wojdyla, ${ }^{1,3}$ David S Pisetsky, ${ }^{1}$ Lisa CriscioneSchreiber, 'Jayanth Doss, ${ }^{1}$ Kai Sun, ${ }^{1}$ Rebecca Sadun, ${ }^{1}$ Megan EB Clowse. ${ }^{1}$ Department of Medicine, Duke University School of Medicine, USA; ${ }^{2}$ Duke Clinical Research Institute, USA; ${ }^{3}$ Durham VA Medical Center, USA

\subsection{6/lupus-2021-lupus21 century.41}

Background The Type 1 \& 2 SLE Model categorizes signs and symptoms as Type 1 (arthritis, nephritis, and rash) and Type 2 (fatigue, brain fog, and widespread pain). It is currently unknown whether Type 2 SLE symptoms vary over time. In this study, we measured longitudinal changes in Type 